\title{
Dermal tolerance and effect on skin hydration of an improved ethanol-based hand gel
}

\author{
Günter Kampf ${ }^{1,2}$, Michael Muscatiello ${ }^{3}$, Dörte Segger 4 \\ ${ }^{1}$ BODE Chemie GmbH \& Co. KG, Scientific Affairs, Melanchthonstrasse 27, 22525 Hamburg, Germany \\ ${ }^{2}$ Institute for Hygiene and Environmental Medicine, Ernst Moritz Arndt University, \\ Walther-Rathenau-Str. 49a, 17489 Greifswald, Germany \\ ${ }^{3}$ Clinical Research Laboratories Inc., 371 Hoes Lane, Piscataway NJ 08854, USA \\ ${ }^{4}$ SIT Skin Investigation and Technology Hamburg GmbH, Dammtorwall 4, 20354 Hamburg, Germany
}

doi:10.3396/ijic.V5i1.003.09

\begin{abstract}
Alcohol-based hand gels have become a standard in hand hygiene in the United States but many of them are significantly less effective than liquid alcohol-based hand disinfectants. An improved ethanol-based gel $(85 \%, w / w)$ with an efficacy equal to liquid products was investigated for dermal tolerance and skin hydrating properties which are essential to achieve a high compliance rate with hand hygiene. For the repetitive occlusive patch test 224 subjects were studied, 213 finished the study. Sterilium Comfort Gel was applied to one site on the back under an occlusive patch during an induction phase (9 applications over 3 weeks) and 2 weeks later to a virgin site on the back during a challenge phase (1 application). Sites were graded for skin reactions using a standardized scale $24 \mathrm{~h}$ after removal of the patches (induction phase and challenge phase) as well as 48 and $72 \mathrm{~h}$ later (challenge phase). To evaluate skin hydrating properties of the gel, treated skin of 23 subjects was compared to untreated skin. The gel was applied twice a day to the forearm for 14 days. Control corneometer values were taken before application of the gel and after 1 and 2 weeks. In the induction phase none of the 213 subjects had a skin reaction. In the challenge phase one subject had a barely perceptible skin reaction at one time point. Relative skin hydration on treated skin in comparison to the untreated control fields was significantly higher after one week by $7.7 \%(p=0.0007$; paired t-test for dependent samples $)$ and after two weeks by $14.1 \%$ ( $<<0.0001)$. The gel did not demonstrate a clinically relevant potential for dermal irritation or sensitization and significantly increased skin hydration after repetitive use and so could enhance compliance with hand hygiene among health care workers.
\end{abstract}

\section{Key words}

alcohol-based hand gel, dermal tolerance, skin hydration

\section{Corresponding author}

Günter Kampf, BODE Chemie GmbH \& Co. KG, Scientific Affairs, Melanchthonstrasse 27, 22525

Hamburg, Germany, Email: guenter.kampf@bode-chemie.be 


\section{Introduction}

Compliance with hand hygiene is a key factor for reducing nosocomial infection rates. ${ }^{1}$ Factors influencing compliance include dermal tolerance ${ }^{2}$ and skin care properties. ${ }^{3}$ The aim of this investigation was to study the dermal tolerance (potential for irritation and sensitisation) of the new gel in a repetitive occlusive patch test as well as its effect on skin hydration.

Hand hygiene is currently undergoing a renaissance in many countries especially since the World Health Organisation has recognized the benefit of alcoholbased hand rubs for the prevention of nosocomial infections. ${ }^{4}$ In the USA the national recommendation has been revised with emphasis on alcohol-based hand rubs. ${ }^{5}$ Alcohol-based liquids have been available for decades, mainly in European countries. Over the last years, alcohol-based hand gels became available as an alternative to liquid preparations. The antimicrobial activity of many hand gels, however, has been demonstrated to be significantly lower in comparison to liquid hand rubs when the total alcohol concentration is below $70 \% .{ }^{6} \mathrm{~A}$ new hand gel has been developed with an ethanol concentration of $85 \%(\mathrm{w} / \mathrm{w})$ which is considered to be safe and effective according to the tentative final monograph for healthcare antiseptic products. ${ }^{7}$ It fulfils the efficacy requirements for the first application for the post contamination treatment of hands with a volume of $2.4 \mathrm{~mL} .^{8}$

\section{Methods}

Hand gel: Sterillium Comfort Gel contains ethanol $(85 \%, w / w)$ as the active ingredient, together with water, a thickening system, skin care components (e.g. glycerol) and a fragrance.

Repetitive occlusive patch test: Subjects between the ages of 20 and 70 were recruited. No individual was included if they had a history of acute or chronic dermatological, medical and/or physical conditions which could interfere with dermal scoring, or treatment with sympathomimetics, antihistamines, non-steroidal anti-inflammatory agents, and/or corticosteroids in the week before the study began. All subjects signed an Informed Consent Form in conformance with 21CFR Part 50: "Protection of Human Subjects".
In order to remove sebum, dead skin cells or any traces of cosmetic or toiletry products the test area was gently wiped using one or two wipes of alcohol-soaked cotton (70\% isopropyl alcohol). This was done only prior to the first induction patch application and the challenge patch application since the subjects were instructed not to use any products on the test sites during the study. The test material $(0.2 \mathrm{ml})$ was allowed to volatilize and was applied under an occlusive patch (occlusive strip with Flexcon, TruMed Technologies Inc., Burnsville, Minnesota, USA) to the upper back between the scapulae. The test material was allowed to remain in direct skin contact for $24 \mathrm{~h}$.

Induction period: Patches were applied to the same site on Mondays, Wednesdays and Fridays for three weeks (nine applications). Patches were removed by the subjects on Tuesdays, Thursdays and Saturdays. The sites were graded by a nurse or scientist trained to score for degrees of erythema and oedema under the supervision of a dermatologist. Grading was done immediately prior to the next product application which was either $24 \mathrm{~h}$ after Tuesday's and Thursday's patch removal or $48 \mathrm{~h}$ after Saturday's patch removal.

Challenge period: After two weeks rest the challenge patches were applied to previously untreated test sites on the back. After $24 \mathrm{~h}$ the test patches were removed by a technician. The test sites were evaluated for dermal reactions immediately after removal of the patches then 48 and $72 \mathrm{~h}$ later.

The sites were graded according to the following scoring system:

$0 \quad$ No visible skin reaction

0.5 Barely perceptible erythema (minimal)

1 Mild erythema (diffuse)

2 Well defined erythema

3 Erythema and oedema

4 Erythema and eodema with vesiculation

Skin hydration study: Female subjects of phototypes I to IV and between the ages of 18 and 70 years were included. Males were excluded because measurement of skin hydration is impaired by hair. Other exclusions 
included: systemic illness, acute skin diseases (e.g. atopic eczema), excess hair, warts, scabs or tattoos on the investigation sites, or if pregnant, breast feeding, diabetic, HIV positive, an alcohol or drug addict or participation in other studies on the forearms 2 weeks prior to study initiation. All subjects signed an Informed Consent Form in conformance with 21CFR Part 50: "Protection of Human Subjects."

About $3 \mu \mathrm{L} / \mathrm{cm}^{2}$ of gel was applied twice a day for 14 days by the volunteers at home according to a protocol from study diary, containing information on times of applications (day, date, morning or evening). Volunteers were instructed to record all product applications. Practical instruction on using the product and the procedures of application was given after the baseline examination. Immediately after the baseline measurements, a product application was performed by volunteers under supervision of the investigator. The investigator checked that the quantity of the applied product, the localization of the application sites, the distribution and the utilization of the product was according to the study protocol. In addition, subjects were provided with printed information concerning the dates for examinations in the Institute and instructions regarding behavior during the study.

The application and investigation sites were on the volar surface of the forearms. For at least seven days before and throughout the study all treatment with leave-on products or use of oily skin cleansing products on the forearms was banned. The product was homogeneously spread over an investigation site of about $7 \times 7 \mathrm{~cm}$ in a quantity of about $150 \mu \mathrm{L}$. The application of the product started immediately after the initial investigation as a controlled application in the institute. In the morning of the $2^{\text {nd }}\left(\right.$ day $8 ; t_{1}$ ) and $3^{\text {rd }}$ day (day $15 ; t_{2}$ ) of measurements, the investigation sites were left untreated. Treatment had been performed on days 1 and 8 as a controlled application in the institute.

Before starting the measurements, the investigation sites were exposed to the indoor climate of the Institute $\left(21.5^{\circ} \mathrm{C} ; 50 \%\right.$ relative humidity) for at least 20 minutes. Skin hydration was measured with the Corneometer CM 825 (Courage \& Khazaka, Cologne, Germany), by placing the probe with low pressure in a vertical manner on to the skin surface. For each application site, six measurements were performed. Data were directly transferred into the computerized study file, classified by study code, code of the volunteer subject, day of the study and code of the investigation site.

The analysis was performed by relating the original data of the product treated investigation sites to the untreated situation and the corresponding starting value. The mean and standard deviation were calculated. Normal distribution of the paired differences was assessed with the Kolmogorov-Smirnov test. Statistical significance was determined using a t-test for dependent samples. A difference was accepted to be statistically significant when the $p$-value was $<0.05$.

\section{Results}

Repetitive occlusive patch test: 224 subjects were enrolled ("intention to treat population"), 213 of them finished the study ("per protocol" population). Eleven discontinued for reasons unrelated to the test preparation. Six subjects did not attend all appointments during the challenge period. Forty-six of the 213 subjects were male $(21.6 \%)$, 167 were female $(78.4 \%)$. The mean age was $44.0 \pm 13.4$ years. None of the 213 had any reaction at any time after the 9 induction applications. $212(99.5 \%)$ had no visible skin reaction 24,48 or $72 \mathrm{~h}$ after the challenge application. One subject had a 0.5 reaction after $48 \mathrm{~h}$ and no visible skin reaction after 24 and $72 \mathrm{~h}$ (Table I).

Skin hydration study: 23 female subjects were recruited, all subjects finished the study. The mean age of the test persons was $45.0 \pm 12.9$ years. The mean skin hydration values before the application of the gel were $34.3 \pm 4.0$ (treated site) and $34.7 \pm 3.7$ (untreated site). Untreated skin areas revealed after 1 week a mean skin hydration value of $34.7 \pm 4.7$ and after 2 weeks a mean value of $34.9 \pm 5.1$. The treated skin areas were found to have a mean skin hydration value of $36.7 \pm 5.7$ after 1 week and $38.9 \pm 5.1$ after 2 weeks. All pair differences were found to be normally distributed after 1 and 2 weeks ( $>0.2$; Kolmogorov-Smirnov test). Differences in skin hydration after relating the data between the treated and untreated test fields (set at 100\%) were significant after 1 week with an increase of $7.7 \%(p=0.0007$; paired t-test for dependant samples) and after 2 weeks with an increase of $14.1 \%(p<0.0001$; Table II). 
Table I: Visual assessment of Sterillium Comfort Gel treated healthy volunteers (per protocol population) during the induction period ( 9 applications over 3 weeks, 213 subjects) and the challenge period ( 1 application on virgin skin site, assessed 1, 2 and 3 days after application, 207 subjects); semi-occlusive test conditions

Scores for irritation in the fields treated with Sterillium Comfort Gel $(n=213)$

\begin{tabular}{lllllll}
\hline $\begin{array}{l}\text { Induction } \\
\text { period: Day }\end{array}$ & Score 0 & Score 0.5 & Score 1 & Score 2 & Score 3 & Score 4 \\
\hline 1 & & & & & & \\
2 & $213 / 213$ & $0 / 213$ & $0 / 213$ & $0 / 213$ & $0 / 213$ & $0 / 213$ \\
3 & $213 / 213$ & $0 / 213$ & $0 / 213$ & $0 / 213$ & $0 / 213$ & $0 / 213$ \\
4 & $213 / 213$ & $0 / 213$ & $0 / 213$ & $0 / 213$ & $0 / 213$ & $0 / 213$ \\
5 & $213 / 213$ & $0 / 213$ & $0 / 213$ & $0 / 213$ & $0 / 213$ & $0 / 213$ \\
6 & $213 / 213$ & $0 / 213$ & $0 / 213$ & $0 / 213$ & $0 / 213$ & $0 / 213$ \\
7 & $213 / 213$ & $0 / 213$ & $0 / 213$ & $0 / 213$ & $0 / 213$ & $0 / 213$ \\
8 & $213 / 213$ & $0 / 213$ & $0 / 213$ & $0 / 213$ & $0 / 213$ & $0 / 213$ \\
9 & $213 / 213$ & $0 / 213$ & $0 / 213$ & $0 / 213$ & $0 / 213$ & $0 / 213$ \\
\hline Challenge & Score 0 & Score 0.5 & Score 1 & Score 2 & Score 3 & Score 4 \\
period: Day & & & & & & \\
\hline 1 & $213 / 213$ & $0 / 213$ & $0 / 213$ & $0 / 213$ & $0 / 213$ & $0 / 213$ \\
2 & $207 / 207$ & $0 / 207$ & $0 / 207$ & $0 / 207$ & $0 / 207$ & $0 / 207$ \\
3 & $206 / 207$ & $1 / 207$ & $0 / 207$ & $0 / 207$ & $0 / 207$ & $0 / 207$ \\
& $207 / 207$ & $0 / 207$ & $0 / 207$ & $0 / 207$ & $0 / 207$ & $0 / 207$
\end{tabular}

Table II: Mean skin hydration values \pm standard deviation of 23 volunteers after 1 and 2 weeks of twice a day use of Sterillium Comfort Gel on the forearm and the corresponding untreated control fields; comparison of relative differences with the paired t-test for dependant probes

\begin{tabular}{lllll} 
& Control & $\begin{array}{l}\text { Sterillium } \\
\text { Comfort Gel }\end{array}$ & Relative difference & p-value \\
\hline Baseline & $34.7 \pm 3.7$ & $34.3 \pm 4.0$ & not applicable & not applicable \\
1 week & $34.7 \pm 4.7$ & $36.7 \pm 5.7$ & $+7.7 \%$ & 0.0007 \\
2 weeks & $34.9 \pm 5.1$ & $38.9 \pm 5.1$ & $+14.1 \%$ & $<0.0001$ \\
\hline
\end{tabular}




\section{Discussion}

Chronic irritative dermatitis in health care workers is an important issue in occupational dermatology. Up to $70 \%$ of health care workers may have occupational hand dermatitis, i.e. those reporting a frequency of hand washing exceeding 35 times per shift. ${ }^{9} \mathrm{~A}$ review of patients presenting with contact dermatitis revealed that almost half (60 of 124) of those with occupational hand dermatitis were health care workers. ${ }^{10}$ Irritant contact dermatitis is found among health care workers almost three times as often as allergic contact dermatitis. ${ }^{11}$ This is partly explained by washing hands even when they are not visibly soiled, or when hand antiseptics are used that lower the skin hydration after repetitive use. $^{12}$

Dermal tolerance is a pre-requisite for acceptance of a hand antiseptic for use among healthcare workers. We were able to show that Sterillium Comfort Gel has no clinically relevant potential for dermal irritation and sensitization, as shown by repetitive occlusive patch test. The design is not based on the actual clinical practice but is, due to the occlusion, even more demanding. Our finding supports previous data which showed that alcohols and well formulated alcoholbased hand rubs have little or no potential for skin irritation. . $^{213-16}$

In clinical practice hands are probably washed quite often when a hand disinfection should be performed so that hands are washed more often than necessary. ${ }^{17}$ This has quite often the effect that the skin becomes dry during work which is the first step towards irritative contact dermatitis. It is therefore desirable to have a hand antiseptic which has the potential for increasing skin hydration especially when occasional hand washing can not be avoided. In that respect alcohol-based hand gels are becoming more popular for the post-contamination treatment of hands which is reinforced by data suggesting that repetitive use of well formulated gels may well increase skin hydration. The gel tested also revealed a significant increase of skin hydration even when applied only twice per day. Based on our study the effect cannot be assigned to specific additives. However there are data to suggest that the effect may be due to glycerol ${ }^{18}$ or panthenol. ${ }^{19}$ Commonly used liquid hand rubs have been reported not to change skin hydration significantly. ${ }^{20}$ Only few alcohol-based hand gels have been tested on the skin for a possible hydration effect. A gel based on $62 \%$ ethanol (Purell, Gojo Industries, Akron, USA) did not increase skin hydration significantly during repetitive use. ${ }^{21}$ A gel based on $85 \%$ ethanol (Sterillium Gel, Bode Chemie $\mathrm{GmbH} \&$ Co. KG, Hamburg, Germany) did increase skin hydration significantly. ${ }^{22}$ Recently a gel based on $75 \%$ isopropanol and $0.5 \%$ chlorhexidine gluconate was also shown to increase skin hydration significantly. ${ }^{23}$

Many attempts have been made to improve compliance with hand hygiene. Skin care is certainly an important factor, and the perception that a product is beneficial to the skin should favor its regular use by health care workers.

\section{Conclusions}

The gel did not demonstrate a clinically relevant potential for dermal irritation or sensitization and significantly increased skin hydration after repetitive use and so could enhance compliance with hand hygiene among health care workers.

\section{Acknowledgements}

The study was funded by Bode Chemie $\mathrm{GmbH} \&$ Co. KG, Hamburg, Germany.

\section{References}

1. Pittet D. Improving compliance with hand hygiene in hospitals. Infection Control and Hospital Epidemiology 2000; 21(6): 381386.

2. Löffler H, Kampf G, Schmermund D, Maibach HI. How irritant is alcohol? British Journal of Dermatology 2007; 157(1): 74-81.

3. Pittet D. Improving adherence to hand hygiene practice: a multidisciplinary approach. Emerging Infectious Diseases 2001; 7(2): 234-240.

4. Anonymous. WHO guidelines on hand hygiene in health care (advanced draft). Geneva: WHO, 2006.

5. Boyce JM, Pittet D. Guideline for hand hygiene in health-care settings. Recommendations of the healthcare infection control practices advisory committee and the HICPAC/SHEA/APIC/ IDSA hand hygiene task force. MMWR 2002; 51: 1-45.

6. Kramer A, Rudolph P, Kampf G, Pittet D. Limited efficacy of alcohol-based hand gels. The Lancet 2002; 359: 1489-1490.

7. Anonymous. Tentative final monograph for health care antiseptic products; proposed rule. Federal Register 1994; 59(116): 31401-31452.

8. Kampf G. How effective are hand antiseptics for the postcontamination treatment of hands when used as recommended? American Journal of Infection Control 2008; 36(5): 356-360.

9. Forrester BG, Roth VS. Hand dermatitis in intensive care units. 
Journal of Occupational and Environmental Medicine 1998; 40(10): 881-885.

10. Wilkinson SM. Effects of infection control measures on skin of health care workers. Community Disease and Public Health 2000; 3(4): 305-306.

11. Nettis E, Colanardi MC, Soccio AL, Ferrannini A, Tursi A. Occupational irritant and allergic contact dermatitis among health care workers. Contact Dermatitis 2002; 46: 101-107.

12. Kampf G, Löffler H. Prevention of irritant contact dermatitis among healthcare workers by using evidence-based hand hygiene practices: a review. Industrial Health 2007; 45(5): 645-652.

13. Lübbe J, Ruffieux C, van Melle G, Perrenoud D. Irritancy of the skin disinfectant n-propanol. Contact Dermatitis 2001; 45: 226-231.

14. Kampf G, Muscatiello M. Dermal tolerance of Sterillium, a propanol-based hand rub. Journal of Hospital Infection 2003; 55(4): 295-298.

15. Kampf G, Wigger-Alberti W, Wilhelm KP. Do atopics tolerate alcohol-based hand rubs? A prospective, controlled, randomized double-blind clinical trial. Acta DermatoVenereologica 2006; 86(2): 140-143.

16. Pedersen LK, Held E, Johansen JD, Agner T. Short-term effects of alcohol-based disinfectant and detergent on skin irritation. Contact Dermatitis 2005; 52: 82-87.
17. Pittet D, Hugonnet $S$, Harbarth $S$, et al. Effectiveness of a hospital-wide programme to improve compliance with hand hygiene. The Lancet 2000; 356: 1307-1312.

18. Jungermann E, Sonntag NOV. Glycerine - a key cosmetic ingredient. New York: Marcel Decker Inc., 1991.

19. Raab W, Kindl U, Daniels R. Pflegekosmetik. Ein Leitfaden. 3rd ed. Stuttgart: Wissenschaftliche Verlagsgesellschaft, 1999.

20. Kramer A, Bernig T, Kampf G. Clinical double-blind trial on the dermal tolerance and user acceptability of six alcohol-based hand disinfectants for hygienic hand disinfection. Journal of Hospital Infection 2002; 51(2): 114-120.

21. Boyce JM, Kelliher S, Vallande N. Skin irritation and dryness associated with two hand-hygiene regimens: soap-and-water hand washing versus hand antisepsis with an alcoholic hand gel. Infection Control and Hospital Epidemiology 2000; 21: 442-448.

22. Kampf G, Muscatiello M, Häntschel D, Rudolf M. Dermal tolerance and effect on skin hydration of a new ethanol-based hand gel. Journal of Hospital Infection 2002; 52(4): 297-301.

23. Traore $\mathrm{O}$, Hugonnet $\mathrm{S}$, Lübbe J, Griffiths W, Pittet D. Liquid versus gel handrub formulation: a prospective intervention study. Critical Care 2007; 11(3): R52. 